\title{
La Biblioteca Digital de la Arquidiócesis de Mérida (Venezuela) y el modelo de metadatos: una propuesta para la difusión de los bienes culturales de la Iglesia católica en la Web
}

\author{
N. Fabiola Rosales \\ Marlene Bauste \\ Universidad de Los Andes (Venezuela) \\ Baltazar E. Porras Cardozo \\ Arquidiócesis de Mérida (Venezuela)
}

\section{Resumen}

La Biblioteca Digital de la Arquidiócesis de Mérida (Venezuela) incorporará para su difusión todos los bienes culturales de la Iglesia merideña. La propuesta se desarrolla en dos grandes etapas. La primera, la definición del modelo de datos/ metadatos que permita describir los diferentes bienes y las relaciones establecidas entre ellos. El objetivo es garantizar la conservación contextual del patrimonio cultural, en este caso de la Arquidiócesis de Mérida, y proponer el uso de estándares de metadatos que garanticen la interoperabilidad de la Biblioteca Digital y aseguren el acceso, en el futuro, a la información y al conocimiento de los bienes culturales de la Iglesia venezolana que se encuentran en formato digital y no digital. En la siguiente etapa se abordará la clasificación de los servicios que se prestarán, enmarcados en la relación que tiene la Iglesia con su comunidad (Gobierno, organizaciones privadas, sector educativo y de investigación), así como el diseño de la interfaz gráfica acorde con la imagen de la Iglesia, la selección y adaptación de la infraestructura tecnológica y, la preservación y difusión de los bienes de la Iglesia.

Palabras clave: Modelo de datos/metadatos. Metadatos. Biblioteca Digital. Iglesia católica venezolana. Arquidiócesis de Mérida. Bienes culturales.

\section{Abstract}

The Digital Library of the Mérida's Archdiocese in Venezuela will incorporate for its diffusion all of the cultural heritage of the Church in this region. The proposal is developed in two stages. First, defining a data/metadata model that allows describing all of the different items and relations established among them. The objective of this stage is to guarantee the contextual conservation of the 
cultural heritage of the Mérida's Archdiocese, and to propose the use metadata standards that assure the interoperability of the Digital Library and assure the access, in the future, to the information and knowledge of the cultural belongings of the Venezuelan Church that are in both digital and no digital formats. The second stage will consist in selecting and classifying the services that the Church will provide to its community, depending on particular relations (i. e. Government, private organizations, education and research), as well as the design of the graphical interface that concur with the image of the Church, the selection and adaptation of the technological infrastructure, and the preservation and diffusion of the Church's patrimony.

Keywords: Data/metadata's model. Metadata. Digital Library. Venezuelan Catholic Church. Mérida's Archdiocese. Cultural heritage.

\section{Introducción}

Uno de los proyectos en ejecución de la Arquidiócesis de Mérida es la creación de la Biblioteca Digital de la Iglesia merideña como medio de almacenamiento, difusión y transferencia de la información sobre los bienes culturales de la Iglesia mediante las tecnologías de la información y de las telecomunicaciones.

En primer lugar es necesario conocer qué se entiende por bienes culturales de la Iglesia católica. El papa Juan Pablo II dirigió un mensaje a los miembros de la primera Asamblea Plenaria de la Pontificia Comisión para los Bienes Culturales de la Iglesia, el 12 de octubre de 1995, mediante el cual estableció que "los bienes culturales de la Iglesia los constituyen los patrimonios artísticos de la pintura, la escultura, la arquitectura, el mosaico y la música, puestos al servicio de la misión de la Iglesia. Además, a estos hay que añadir los libros contenidos en las bibliotecas eclesiásticas y los archivos de las comunidades eclesiales. En fin, pertenecen a este ámbito las obras literarias, teatrales y cinematográficas producidas por los medios de comunicación social" (Arquidiócesis de Mérida, 2003, p. 11).

La posibilidad de ofrecer esta información en la Web ha sido preocupación de las autoridades eclesiásticas, quienes desde finales de la década de los años noventa se plantearon la creación de la Red de Información de la Iglesia Católica de Mérida.

Este interés condujo a la investigación que se adelanta hoy en día en la Arquidiócesis sobre biblioteca digital para decidir el modelo que se ha de seguir. Se llegó al acuerdo de que el mismo debía definirse con una intención precisa, con reglas de uso para la descripción de los bienes culturales, considerando entre ellas la semántica para el significado de cada campo que se tiene que describir y el modo de usarlo, las reglas de contenido que determinan el o los valores del campo y la sintaxis.

En la actualidad, la Arquidiócesis de Mérida cuenta con repositorios físicos, un catálogo electrónico que referencia solo una parte de su patrimonio bibliográ-

Scire. $14: 2$ (jul.-dic. 2008) 131-149. ISSN 1135-3716. 
fico y archivístico, así como varios catálogos impresos de registros documentales y museísticos; estos catálogos se usaron como base para identificar todos los campos y atributos que conformarían el modelo de metadatos.

La definición del modelo de datos/metadatos que permita describir los patrimonios artísticos de la pintura, la escultura, la arquitectura y el mosaico para la Biblioteca Digital de la Iglesia merideña a partir del análisis y comparación de los esquemas existentes es el producto esperado de este trabajo. Se posterga el tratamiento de los otros bienes, como las bibliotecas y los archivos eclesiales, para una siguiente etapa del proyecto.

\section{La Iglesia católica y su patrimonio: inventario y catalogación}

Es determinante el interés de la Iglesia por registrar de manera ordenada la información sobre su patrimonio histórico-artístico, constituido por los bienes culturales. El inventario y la consecuente catalogación constituyen la metodología para alcanzar este fin. De esta manera, "el inventario pasa a formar parte de las disposiciones del Código de Derecho Canónico (1983), en el cual se prescribe la obligación de redactar un inventario exacto y detallado [...] de los bienes inmuebles, de los bienes muebles, tanto preciosos como pertenecientes de algún modo al patrimonio cultural y de cualesquiera otros con la descripción y tasación de los mismos" (Arquidiócesis de Mérida, 2003, p. 14), lo que convierte a la Iglesia católica en la primera institución pública que reglamenta, estableciendo sus propias leyes, la creación, la conservación y la valoración de sus bienes culturales.

Se deriva entonces la necesidad de una descripción más detallada del ingente patrimonio de la Iglesia, de forma integral, tomando en consideración todos los elementos y en su contexto. Con ello la Iglesia persigue asegurar el resguardo y valoración eclesial de los bienes culturales y generar una guía como modelo para las iglesias particulares en la realización de sus propios inventarios.

La Arquidiócesis de Mérida ha desarrollado su metodología, con procedimientos y formatos apegados a los estándares internacionales para el registro del inventario y la catalogación de sus bienes culturales.

\subsection{El inventario y la catalogación de los bienes culturales de la Iglesia}

Llama la atención la manera en que la Iglesia conceptualiza las actividades de inventario y de catalogación como procesos cognoscitivos y de valoración de su patrimonio. El inventario como un proceso de reconocimiento de las características extrínsecas de los objetos y la catalogación como la descripción integral de los bienes y sus relaciones intrínsecas con el entorno o contexto.

Así, la Circular de la Pontificia Comisión para los Bienes Culturales de la Iglesia sienta las bases del inventario-catalogación al definir el objeto, el método y los objetivos de este binomio, dada la singularidad del patrimonio histórico-artístico 
de la Iglesia; por tanto no pueden ser reconocidos, tutelados y valorados a través de un proceso sencillo de registro.

\section{De esta manera, la Circular define}

el objeto material del inventario-catalogación como el bien cultural de interés religioso en cuanto a objeto, es decir, en cuanto obra producida por el hombre, visible, mesurable, deteriorable. [...] De esta definición quedan excluidos los bienes culturales, es decir, las obras no producidas por el hombre, y el conjunto de los bienes culturales no materiales como la lengua, las costumbres, los mitos los modelos de comportamiento. (Arquidiócesis de Mérida, 2003, p. 22)

Siguiendo la Carta Circular (Arquidiócesis de Mérida, 2003, p. 22), los bienes objeto de inventario y catalogación se clasifican en bienes inmuebles y bienes muebles. Entre los primeros se encuentran, por ejemplo, los edificios-iglesias, monasterios, conventos, palacios episcopales, casas parroquiales y los complejos educativos y caritativos. Entre los bienes muebles están las pinturas, las esculturas, los ornamentos litúrgicos, los instrumentos musicales, etcétera. Los archivos y los libros, como bienes documentales, son objeto de diversos tratamientos metodológicos debido al carácter antropológico, cultural y ambiental de los mismos.

A efectos de este trabajo fue importante tener en cuenta, en primera instancia, lo expresado por la Pontificia Comisión para los Bienes Culturales de la Iglesia en cuanto al carácter del inventario-catalogación de los bienes culturales:

el inventario catalogación no es una simple operación enumerativa, sino una selección razonada de información, sobre la base de un particular cuadro ideológico y epistemológico de referencia. Por lo tanto, ya a partir de los datos encontrados, se debe madurar la intención de tomar en consideración el valor histórico-artístico, lo específico eclesial, la unidad contextual, la pertinencia jurídica y el estado material de tales bienes, con el fin de poner en sintonía la labor de reconocimiento con el sensus eclessiae. (Arquidiócesis de Mérida, 2003, p. 22)

La propuesta que ofrecemos se circunscribe al patrimonio artístico de pinturas, esculturas, arquitectura y mosaicos existentes en la Iglesia merideña.

La Carta Circular (Arquidiócesis de Mérida, 2003, p. 23) señala que el método usado en el trabajo de inventario-catalogación involucra tres fases que encontramos en las disciplinas histórico-artísticas; a saber: 1) fase heurística o de individualización; 2) fase analítica o de descripción; y 3) fase de la síntesis o de ordenación y organización final del trabajo para la obtención de los resultados.

Los objetivos del inventario-catalogación están asociados a) al conocimiento preciso del patrimonio histórico artístico de la Iglesia "en su unitaria globalidad, en la complejidad de las relaciones existentes entre los objetos que lo componen, en su indisoluble relación con la historia y el territorio"; $b$ ) a la salvaguardia concebida desde cuatro puntos de vista: el eclesial, el técnico, el administrativo y el de la seguridad (el primero determina la naturaleza religiosa de los bienes; el segun-

Scire. 14 : 2 (jul.-dic. 2008) 131-149. ISSN 1135-3716. 
do se refiere a las particularidades del bien y su relación con el contexto histórico; el tercero requiere el conocimiento de la propiedad, y el cuarto se compromete con la generación del registro conforme con los entes responsables); y c) la valoración de esos bienes, con lo que se busca el reconocimiento, el respeto y el disfrute de ese patrimonio en su identidad eclesial, cultural, social, histórica y artística.

La cristalización del trabajo del inventario-catalogación es la ficha catalográfica, la cual recoge de manera sintética la información producto del análisis del objeto en estudio. Las características de la ficha las determina el tipo de objeto que se describe, para lo cual se cuenta con normas o estándares internacionales que orientan la consecución de un modelo; la incorporación de los sistemas automatizados para los procesos de inventario-catalogación coadyuvan a la creación de catálogos ampliando el acceso para los estudiosos y los interesados en los bienes culturales de la Iglesia, sin distingos ideológicos o religiosos.

La ficha catalográfica está compuesta de elementos denominados campos para la descripción del o de los objetos. La Circular de la Pontificia Comisión para los Bienes Culturales de la Iglesia (Arquidiócesis de Mérida, 2003, p. 37) define para el patrimonio histórico-artístico los campos objeto, material, medidas, localidad, propiedad y estado de conservación, con el propósito de precisar el objeto y su contexto. La finalidad es mantener el contexto originario o lograr su reconstrucción para la valorización religiosa de los bienes dispersos y promover el disfrute de las obras en su ubicación espacial.

A continuación se describen los elementos básicos que componen la ficha catalográfica de tipo analítico-sintética y sus características:

1. Signatura topográfica: código numérico o alfanumérico que individualiza el objeto de descripción y lo ubica en un contexto temático o de lugar.

2. Vocablos normalizados o palabras claves: se utilizan los tesauros especializados para la definición de los términos.

3. Descripción del objeto o bien cultural en cuanto forma, tamaño, tipo en su género y estado de conservación, incluyendo como principal dato al autor, en el caso de que exista, entre otros elementos que considere la unidad catalogadora.

4. Institución y/o ubicación tutelar del bien.

5. Descripción visual de bien: se especifica si se trata de un documento, una fotografía, una escultura, planos, etcétera.

Así, la catalogación es un proceso técnico de organización del conocimiento conducente a la valorización y salvaguardia de los bienes culturales, requerido para la gestión y preservación de los mismos. 


\subsection{La Iglesia merideña y su patrimonio: un modelo de organización}

La vida de las iglesias particulares gravita en torno a sus obispos. Por razones teológicas y prácticas su sello personal marca el período en que les toca gobernar una diócesis.

La Iglesia merideña está representada por la Arquidiócesis de Mérida, anterior Diócesis de Mérida de Maracaibo, erigida por Su Santidad Pío VI por la bula Magnitudo Divinae Bonitatis, de fecha 16 de febrero de de 1778.

Geográficamente se extiende por gran parte del territorio del estado de Mérida, en una superficie aproximada de 8109 kilómetros cuadrados y con una población estimada al 17 de noviembre de 2003 de 601570 habitantes.

La Arquidiócesis cuenta con una estructura organizacional compleja, la cual comprende 1 curia arzobispal, 5 vicarías o zonas pastorales, 61 parroquias, 14 iglesias filiales y 2 vicarías parroquiales (con un número aproximado de 600 templos y/o capillas). Incluye, además de las entidades administrativas, parroquiales, conventos y congregaciones, unidades académicas, de investigación, de servicios sociales, de salud y de medios de comunicación. Entre ellos hay algunos de singular importancia, tales como el Seminario San Buenaventura (1785), el Archivo (1905) y el Museo Arquidiocesano (1909) y el Centro de Estudios Teológicos Juan Pablo II (1986).

Históricamente ha contado con 10 obispos desde 1782 hasta 1923 y 6 arzobispos desde 1923 hasta la fecha. El actual titular es el excelentísimo monseñor Baltazar E. Porras Cardozo, desde el 5 de diciembre de 1991.

La Arquidiócesis cuenta actualmente con 1 arzobispo, 1 obispo auxiliar y 93 sacerdotes diocesanos, de los cuales 7 están jubilados, 8 trabajando por convenios en otros países, 8 estudiando en el exterior y 1 en proceso de excardinación.

Respecto a comunidades religiosas masculinas existen 1 orden monástica, 4 órdenes mendicantes, 1 orden de clérigos regulares, 5 congregaciones clericales, 1 sociedad de vida apostólica y 1 congregación religiosa laical. En cuanto a las femeninas, hay 1 orden religiosa monástica, 27 congregaciones religiosas femeninas y 3 institutos seculares.

La Arquidiócesis ha realizado un trabajo de organización, inventario y catalogación del patrimonio documental y museístico, así como también de su biblioteca de libros antiguos, parte de la biblioteca de monseñor Porras, y ha concluido el registro e inventario de la colección del Museo Arquidiocesano. Este trabajo se reseña en diversas publicaciones como los Boletines de la Arquidiócesis y del Archivo Arquidiocesano de Mérida en sus distintas ediciones, el Directorio de la Arquidiócesis de Mérida en sus versiones impresa y electrónica, y en publicaciones tales como la serie "Investigaciones" y las guías didácticas del Museo.

El proceso de recolección de datos e información para la realización de los inventarios y la catalogación de los distintos materiales de la Iglesia merideña (do- 
cumentos, objetos y libros) implicó la generación de los correspondientes formatos, que se han convertido en la fuente primaria de información para la definición del modelo de metadatos. A continuación se muestran los distintos formatos.

Ficha de inventario del Museo. Tiene un formato de dos caras, compuestas ambas de secciones y subsecciones. La cara A presenta seis de estas. La primera tiene tres elementos para la identificación: número de la pieza, número anterior y código del bien cultural. La siguiente sección corresponde a la descripción y contiene dos subsecciones: clasificación genérica y dimensiones en centímetros. Estas recogen la información sobre el área y la sección, el nombre del objeto, el del autor y las características físicas de la obra.

En la sección de datos técnicos se registra la información sobre procedencia; cultura, tradición o grupo étnico; época, estilo, movimiento o escuela; valor o moneda; responsable del avalúo; fecha, y propietario original. En la columna derecha se contempla el registro de los datos de la fotografía del bien en cuestión, como el número de rollo y el número de negativo. Se reserva el espacio para la ubicación de la fotografía. La siguiente sección se refiere al estado de conservación y las condiciones de integridad. Por último, se anotan las referencias de donde se tomó la información para la descripción.

La cara B se compone de 4 secciones. En la inicial, de observaciones, se registran los datos sobre inscripciones, uso, historia y otras consideraciones. Sigue la sección sobre fuente, forma y fecha de ingreso en la colección. Seguidamente
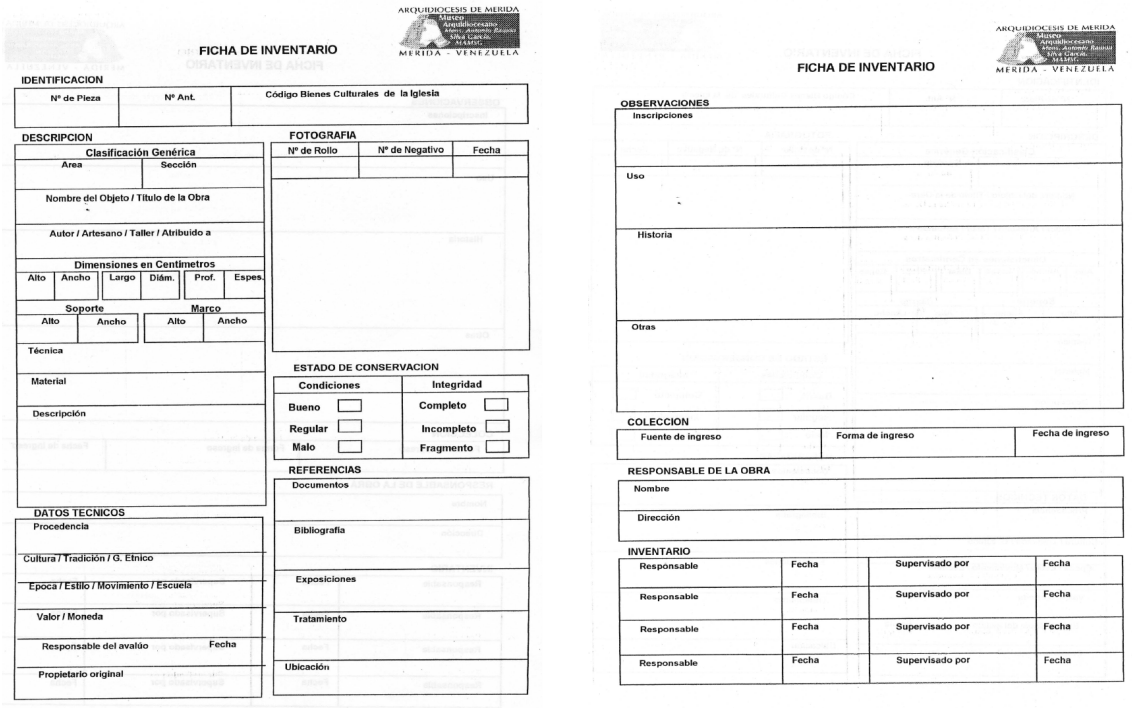

Figura 1. Ficha de inventario del Museo (fuente: Museo Arquidiocesano de Mérida).

Scire. $14: 2$ (jul.-dic. 2008) 131-149. ISSN 1135-3716. 
se contemplan los datos sobre los responsables de la obra y se termina con la información sobre los responsables y fechas del inventario.

Ficha de inventario de libros. Planilla de una sola cara en la que se diferencian siete campos de una sección compuesta de 19 columnas y de tantas filas como libros existan en la colección.

Entre las sietes primeras secciones encontramos los campos correspondientes al encuestador o realizador del inventario, fecha, número de la hoja, nombre de la institución, lugar, estado y dirección. Continúa el área conformada por las 19 columnas donde se recogen los datos sobre número del ejemplar, año, identificación, número de partidas, número de documento, folio, página, encuadernación (con cinco columnas: original, cartón, cuero, papel, tela), conservación (con tres: buena, regular, mala), escritura (con tres: manual, impresa, meconografiada) y, por último, observaciones.

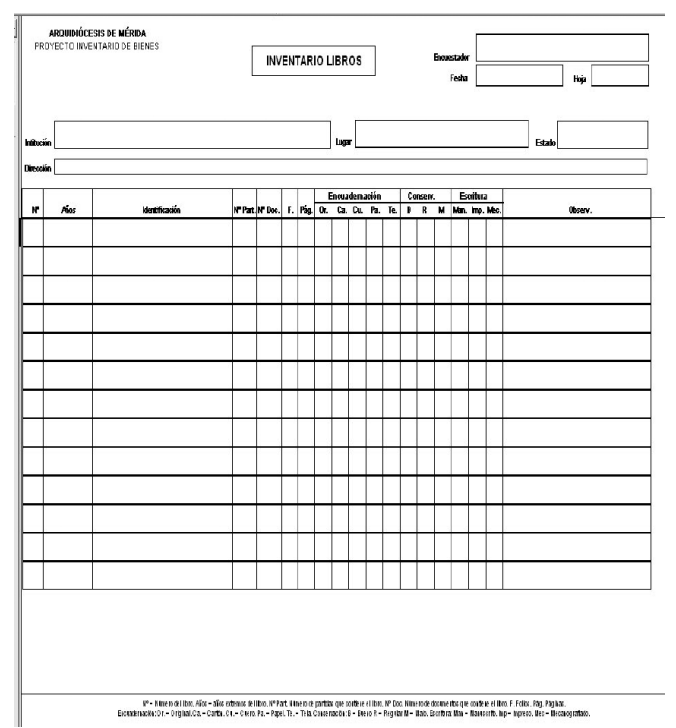

Figura 2. Ficha de inventario de libros (fuente: Archivo Arquidiocesano de Mérida).

Ficha catalográfica de materiales bibliográficos. Se basa en las Reglas de Catalogación Angloamericanas (AACR) y en MARC para la construcción de su OPAC o catálogo público automatizado (http://arqmrd.no-ip.org/arqmrd/). Está conformada por 20 campos de descripción bibliográfica, con la aplicación del segundo nivel de descripción para los libros de reciente edición y del tercer nivel de descripción para los libros antiguos. 
0XX 1 XX $|2 X X| 3 X X|4 X X| 5 X X|6 X X| 7 X X|8 X X| 9 X X|X X X|$ ficha marc

960925625099609|'23eueuiruiz nam9aB

008 9609256????????????????????????????????

020 - \$a 980-221-810-3

039 V- \$a 2 \$b 3 \$c 3 \$d 3 \$e 3

040 - $\quad \$ a$ eui $\$ c$ eui

099 - \$a BX4705 \$b C3P6

100 \#\# \$a Porras Cardozo, Baltazar Enrique \$d 1944-

245 \#\# \$a Torrijos y Espinoza \$b dos breves episcopados merideños \$c Baltazar E. Porras Cardozo

260 - \$a Mérida \$b Talleres Gráficos Universitarios \$c 1994

300 - \$a $197 p$

490 U- \$a Colección el Archivo \$v 5

504 - \$a Incluye bibliografía

60021 \$a Cándido Torrijos y Rigueiros, Manuel Fray \$d 1735-1794

60021 \$a Espinosa y Lorenzo, Antonio Ramón de, Fray \$d 1735-1800

650 -C \$a Obispos \$z Mérida \$x Biografías

61020 \$a Iglesia católica \$z Mérida

850 - \$a eap \$a eam \$a ead

Figura 3. Ficha catalográfica de materiales bibliográficos.

\begin{tabular}{|r|l|}
\hline Cota: & BX4705 C3P6 \\
\hline Autor: & Porras Cardozo, Baltazar Enrique, 1944- \\
\hline Título: & $\begin{array}{l}\text { Torrijos y Espinoza: dos breves episcopados merideños / } \\
\text { Baltazar E. Porras Cardozo }\end{array}$ \\
\hline Publicación: & Mérida: Talleres Gráficos Universitarios, 1994 \\
\hline Descripción: & $197 \mathrm{p}$ \\
\hline Tipo de material: & \\
\hline Biblioteca: & $\begin{array}{l}\text { Biblioteca Monseñor Porras (siglas: eap) } \\
\text { Biblioteca Museo Arquidiocesano (siglas: eam) } \\
\text { Biblioteca Dirección del Archivo (siglas: ead) }\end{array}$ \\
\hline
\end{tabular}

Figura 4. Visualización del registro del OPAC.

\section{Metadatos, una revisión documental}

En esta sección, más que ofrecer un estudio profundo del origen y concepto de los metadatos, materia ampliamente estudiada y difundida en medios impresos y electrónicos, nos dedicaremos al estudio y comparación de esquemas de metadatos existentes para la descripción de bienes culturales, con el propósito de definir el modelo más ajustado para la Iglesia merideña. No obstante, es pertinente partir del enunciado del concepto de metadatos de algunos autores.

La abundancia de información y su permanente crecimiento en la Web llevó a los investigadores a la necesidad de crear mecanismos y herramientas que Scire. $14: 2$ (jul.-dic. 2008) 131-149. ISSN 1135-3716. 
permitieran la búsqueda y recuperación de información de manera oportuna y eficiente. Basados en los métodos tradicionales de descripción, catalogación y clasificación, produjeron esquemas o estándares para el medio electrónico. Los esquemas metadatos son el resultado de estos esfuerzos.

Desde los años sesenta hasta la fecha muchos autores han definido el concepto de metadatos partiendo de la acepción clásica de que no son más que datos sobre datos. Entre ellos destacamos a la investigadora María Jesús Lamarca (2006), quien en su tesis doctoral titulada Hipertexto: el nuevo concepto de documento en la cultura de la imagen parte de esta acepción haciendo referencia a un sentido más amplio o más preciso del mismo. En sentido amplio pone de ejemplo los catálogos bibliográficos como metadatos que ofrecen características sobre el tipo de información del elemento — libro — que se describe, para lo cual se utilizan las Reglas de Catalogación Angloamericanas; para la transferencia y recuperación automática de esa información se utiliza el formato MARC (MAchine Readable Cataloging). Cada registro es una ficha catalográfica y se convierte en un conjunto de metadatos, dado que no solo ofrece información básica sobre la obra y su autor, sino que la relaciona con otras obras del mismo autor o de similar contenido. En sentido restringido, los metadatos son usados exclusivamente para el entorno digital y en red, dadas sus características intrínsecas de uso para la localización, identificación y descripción de recursos electrónicos.

Senso y De la Rosa Piñero (2003), en su artículo "El concepto de metadato. Algo más que descripción de recursos electrónicos", ofrecen una definición a partir de la revisión y análisis de varias propuestas, y concluyen que "metadato es toda aquella información descriptiva sobre el contexto, calidad, condición o características de un recurso, dato u objeto que tiene la finalidad de facilitar su recuperación, autentificación, evaluación, preservación o interoperatividad".

A partir de esta definición nos queda claro que el desarrollo de un esquema de metadatos es fundamental en un proyecto de biblioteca digital para garantizar la accesibilidad, la difusión, la autenticidad, la optimización del tiempo de respuesta en la búsqueda, la preservación del objeto original y la transferencia de la información.

Algunos autores han clasificado los metadatos de acuerdo a su función, forma y estructuración, y se encuentran incluso de metadatos según las personas o instituciones que lo crean. Otros han agrupado estas tipologías de metadatos con base en su función. Para efectos de nuestro trabajo haremos referencia a la propuesta de Gilliland (1998) respecto a la función, por ser la más completa y usada en esta disciplina:

Metadatos administrativos. Dan información necesaria para la gestión y administración de los recursos de información. Este tipo de metadatos incluye número de registro, fecha de creación del registro, fecha de la última modificación, identificación del creador/ revisor del registro, lengua del registro, notas, relación de este registro con otro(s) y gestión de derechos, entre otros.

Scire. $14: 2$ (jul.-dic. 2008) 131-149. ISSN 1135-3716. 
Metadatos descriptivos. Se utilizan para describir las propiedades físicas e intelectuales o el contenido del recurso de información con elementos tales como títulos, autores, colaboradores, fecha, editor, identificador del recurso (URL) e índices, entre otros. Este tipo de metadatos se usa para indizar e identificar el recurso.

Metadatos de preservación. Se emplean para dar información que permita salvaguardar los recursos de información. Se incluyen datos como resolución, formato, equipo y software usados para crear la imagen digital, entre otros.

Metadatos técnicos. Se refieren a cómo funcionan los sistemas o el comportamiento de los metadatos. Dan información sobre el hardaware y el software que se utilizan para convertir un objeto a formato digital, el almacenamiento, la visualización, la autenticación y los datos de seguridad, entre otros.

Metadatos de uso. Se refieren al nivel y el tipo de uso del recurso. Aportan información sobre versiones y reutilización del contenido del recurso.

Por otra parte, existen diversas iniciativas de esquemas de metadatos en atención a los propósitos, ya sean generales o específicos:

Metadatos de propósito general. Están dirigidos a la descripción de cualquier tipo de objeto digital. La iniciativa más conocida y que ha tenido mayor éxito es el Dublin Core.

Metadatos de propósito específico. Van dirigidos a un área, ámbito o dominio específico; entre ellos tenemos los sectores de educación, geografía, industria, Gobierno y patrimonio cultural, literario y artístico, y audiovisual, entre otros.

En el número 3 de Metadata Watch Report (Dekkers, 2000) encontramos una revisión de los esquemas de metadatos más conocidos según su finalidad, para educación, patrimonio cultural, geográfico, literario y artístico, entre otros. En la tabla I se presentan algunos esquemas de metadatos para diferentes propósitos.

\begin{tabular}{|c|c|c|c|c|c|}
\hline Esquema de metadatos & General & Educación & Geográfico & Patrim. cultural & Lit. y artístico \\
\hline Dublin Core & $x$ & & & & \\
\hline MET & $x$ & & & & \\
\hline MODS & $x$ & & & & \\
\hline ARIADNE & & $x$ & & & \\
\hline DC-EDUCATION & & $x$ & & & \\
\hline EDNA & & $x$ & & & \\
\hline EVN & & $x$ & & & \\
\hline FGDC & & & $x$ & & \\
\hline ANZLIC & & & $x$ & & \\
\hline CDWA Lite & & & & $x$ & \\
\hline REACH & & & & $x$ & \\
\hline VRA Core & & & & $x$ & \\
\hline EAD & & & & $x$ & \\
\hline TEI & & & & & $x$ \\
\hline
\end{tabular}

Tabla I. Esquemas de metadatos según su propósito.

Scire. $14: 2$ (jul.-dic. 2008) 131-149. ISSN 1135-3716. 


\subsection{Esquemas objeto de estudio}

En este trabajo solo estudiaremos la iniciativa Dublin Core y las definidas para el patrimonio cultural, tales como CDWA Lite, REACH y VRA Core, por su uso relevante en los desarrollos de bibliotecas digitales por parte de las instituciones internacionales más reconocidas en el mundo cultural con el fin de definir en primer lugar el esquema de metadatos para el Museo Arquidiocesano como punto de partida para la creación del esquema de metadatos para todos los bienes culturales de la Iglesia católica de Mérida.

\subsubsection{Dublin Core (DC) (2006; Dekkers, 2000)}

El estándar de metadatos Dublin Core (DC) o DCMI es un esfuerzo internacional e interdisciplinario para describir recursos electrónicos y facilitar la recuperación de información en la Web. Tiene gran aceptación, se utiliza en metamodelos de descripción de información como el Resource Description Framework (RDF), y es uno de los más usados en las bibliotecas digitales. Dublin Core posee 15 elementos clasificados en tres grupos: de contenidos, de propiedad intelectual y de instanciación. Los elementos tienen nombres descriptivos que explicitan su significado semántico; pueden ser obligatorios, opcionales y repetibles.

\subsubsection{CDWA (Categories for the Description of Works of Art)} (Getty Trust, 2005-2006)

El propósito de este esquema es describir un formato para obras de arte y material cultural. Fue diseñado para las comunidades que manejan información artística (museos y archivos). Está formado por 22 elementos clasificados en dos grupos: elementos descriptivos y elementos administrativos.

\subsubsection{REACH element set for shared description of museum objects (RLG, 2006)}

Este esquema resulta de utilidad para museos, instituciones que trabajan con patrimonio cultural y subastadores, entre otros. Se ofrece como un modelo para avanzar en un estándar más general a la hora de compartir información entre museos. Está conformado por 20 elementos.

\subsubsection{VRA Core Categories (Visual Resources Association) (VRA, 2006)}

El VRA Core Categories fue diseñado para compartir información entre colecciones de recursos visuales. Posee un conjunto de 17 elementos que pueden ser aplicados tantas veces como sea necesario para crear registros que describan trabajos culturales así como las imágenes que los documentan.

En la tabla II se muestran los esquemas de metadatos y los conjuntos de elementos que los conforman. 


\begin{tabular}{|l|l|l|l|}
\hline Dublin Core & CDWA & VRA Core & REACH \\
\hline Title & Object/work type wrapper & Title & Object name/title \\
\hline Creator & Title wrapper & Creator & Creator/maker \\
\hline Subject & Display creator & Subject & Subject matter \\
\hline Description & Indexing creator wrapper & Description & Dimensions \\
\hline Publisher & Display measurement & Record type & Style/period/group/movement/school \\
\hline Contributor & Indexing measurements wrapper & Measurement & Nacionality/culture of creator/maker \\
\hline Date & Display materials/techniques & Date & Date of creation / date range \\
\hline Type & Indexing materials/technique wrapper & Type & Type object \\
\hline Format & Display state/edition wrapper & Material & Medium/materials \\
\hline Identifier & Style wrapper & ID number & Electronic location \& access \\
\hline Source & Culture wrapper & Source & Notes \\
\hline Language & Display creation date & Culture & Lenguaje \\
\hline Relation & Indexing dates wrapper & Relation & Related objets \\
\hline Coverage & Location/repository wrapper & Location & Place of origin/discovery \\
\hline Rights & Subject indexing wrapper & Rights & Techniques/process \\
\hline & Class wrapper & Style period & Current owner \\
\hline & Description / descriptive note wrapper & Technique & Current repository name \\
\hline & Inscriptions wrapper & & Current repository place \\
\hline & Related works wrapper & & Current object ID number \\
\hline & Rights for work & & Provenance \\
\hline & Record wrapper & & \\
\hline
\end{tabular}

Tabla II. Conjuntos de elementos de los esquemas de metadatos en estudio.

\subsection{Definición del modelo de metadatos para la Iglesia católica merideña}

La definición del esquema de metadatos implicó una revisión exhaustiva sobre los temas relativos a inventario y catalogación de bienes culturales de la Iglesia católica, así como en lo referente a metadatos. La investigación bibliográfica no mostró evidencia de la existencia de un esquema o propuesta de metadatos para los bienes culturales de la Iglesia católica. Sin embargo, hay esquemas para la descripción de bienes culturales, tales como CDWA, REACH y VRA Core, entre otros. Encontramos proyectos sobre el patrimonio cultural, como el North Carolina ECHO: Exploring Cultural Heritage Online (Wisser, 2006), patrocinado por Duke University Libraries y State Library of North Carolina, que ofrecen información completa sobre esta experiencia.

El análisis producto de esta revisión, en el conocimiento de las exigencias de la Iglesia católica en cuanto al registro e inventario de los bienes culturales para el

Scire. 14 : 2 (jul.-dic. 2008) 131-149. ISSN 1135-3716. 
culto, la evangelización y la difusión de la cultura de los pueblos, dio como resultado la identificación de los elementos que debe contener el esquema de metadatos para el patrimonio museístico y edificado de la Iglesia merideña, los cuales se muestran en la tabla III.

\begin{tabular}{|c|c|c|}
\hline N. ${ }^{\circ}$ & Elemento & Descripción \\
\hline 1 & Título & Nombre dado a la obra de arte, arquitectura o bien cultural \\
\hline 2 & Creador/contribuidor & $\begin{array}{l}\text { Nombre, información biográfica breve y rol del creador en el } \\
\text { diseño y producción del trabajo. Se incluyen los restauradores }\end{array}$ \\
\hline 3 & $\begin{array}{l}\text { Datos de indización } \\
\text { del creador/contribuidor }\end{array}$ & $\begin{array}{l}\text { Nombre del creador, nacionalidad, fecha de nacimiento y } \\
\text { muerte, sexo, rol }\end{array}$ \\
\hline 4 & Tipo & $\begin{array}{l}\text { Término que identifica el objeto o trabajo que está siendo } \\
\text { descrito }\end{array}$ \\
\hline 5 & Materia/área & Materia o disciplina donde se ubica del trabajo (índice) \\
\hline 6 & Dimensiones & $\begin{array}{l}\text { Información acerca de las dimensiones, tamaño o escala del } \\
\text { trabajo }\end{array}$ \\
\hline 7 & Material/técnica & $\begin{array}{l}\text { Materiales y técnicas utilizados para la elaboración del trabajo } \\
\text { (índice) }\end{array}$ \\
\hline 8 & Estado de conservación & Descripción del estado de conservación del trabajo \\
\hline 9 & Estilo histórico & $\begin{array}{l}\text { Términos que identifican el nombre, estilo definido, período } \\
\text { histórico o artístico, movimiento, grupo o escuela, cuyas } \\
\text { características están representadas en el trabajo que está } \\
\text { siendo catalogado }\end{array}$ \\
\hline 10 & Cultura & Nombre de la cultura desde la cual se ha originado el trabajo \\
\hline 11 & Fechas asociadas & $\begin{array}{l}\text { Fechas asociadas al diseño, ejecución, modificación o } \\
\text { alteración, restauración, destrucción, descubrimiento (índice) }\end{array}$ \\
\hline 12 & Localización & $\begin{array}{l}\text { Nombre y localización geográfica del repositorio responsable } \\
\text { del trabajo; para monumentos y arquitectura, localización } \\
\text { geográfica del trabajo }\end{array}$ \\
\hline 13 & Descripción/referencia & Notas descriptivas y sus fuentes \\
\hline 14 & Inscripciones & $\begin{array}{l}\text { Descripción o trascripción de alguna identificación física de } \\
\text { letras, anotaciones, textos, marcas o etiquetas que estén } \\
\text { adheridas, adosadas, estampadas al trabajo }\end{array}$ \\
\hline 15 & Derechos para reproducción & $\begin{array}{l}\text { Información acerca del manejo de los derechos; puede incluir } \\
\text { copyright y otras declaraciones de propiedad intelectual }\end{array}$ \\
\hline 16 & URL & Dirección electrónica del recurso \\
\hline 17 & Trabajos asociados & Trabajos relacionados con la obra que se está describiendo \\
\hline
\end{tabular}

Tabla III. Modelo de metadatos para el patrimonio artístico de la Iglesia católica merideña. 
Debemos destacar que para la comparación se tomaron en cuenta, además de los elementos de descripción general, los calificadores y los subelementos de los esquemas en estudio. Al comparar el modelo propuesto para la Iglesia merideña (tabla III) con los estándares existentes se observó una correspondencia completa con el esquema de metadatos CDWA, como se muestra en la tabla IV. Este resultado nos permite inferir que el modelo CDWA puede ser una opción válida para

\begin{tabular}{|c|c|c|c|c|}
\hline MICM & Dublin Core & CDWA & VRA Core & REACH \\
\hline Título & Title & Title wrapper & Title & Object name/title \\
\hline Creador/contribuidor & Creator & Display creator & Creator & Creator/maker \\
\hline $\begin{array}{l}\text { Datos de indización } \\
\text { del creador/contribuidor }\end{array}$ & & Indexing creator wrapper & & \\
\hline Tipo & Type & Object/work type wrapper & Type & Type object \\
\hline Materia/área & Subject & Subject indexing wrapper & Subject & Subject matter \\
\hline Dimensiones & Format & Display measurement & Measurement & Dimensions \\
\hline Material/técnica & & Display materials/techniques & $\begin{array}{l}\text { Technique, } \\
\text { material }\end{array}$ & $\begin{array}{l}\text { Medium/materials, } \\
\text { techniques/process }\end{array}$ \\
\hline Estado de conservación & & Display state/edition wrapper & & \\
\hline Estilo histórico & & Style wrapper & Style, period & $\begin{array}{l}\text { Style/period/group/ } \\
\text { movement/school }\end{array}$ \\
\hline Cultura & & Culture wrapper & Culture & $\begin{array}{l}\text { Nacionality/culture of } \\
\text { creator/maker }\end{array}$ \\
\hline Fechas asociadas & Date & Display creation date & Date & Date of creation/date range \\
\hline Localización & Coverage & Location/repository wrapper & & Current repository place \\
\hline Descripción/referencia & Description & $\begin{array}{l}\text { Description / descriptive } \\
\text { note wrapper }\end{array}$ & Description & Notes \\
\hline Inscripciones & & Inscriptions wrapper & & \\
\hline $\begin{array}{l}\text { Derechos para } \\
\text { reproducción }\end{array}$ & Rights & Rights for work & Rights & \\
\hline URL & Identifier & & ID number & Electronic location \\
\hline \multirow[t]{7}{*}{ Trabajos asociados } & Relation & Related works wrapper & & Related object \\
\hline & Publisher & Class wrapper & Relation & Current owner \\
\hline & Contributor & Record wrapper & Record type & Current repository name \\
\hline & Source & Resource wrapper & & Current object ID number \\
\hline & Language & $\begin{array}{l}\text { Indexing measurements } \\
\text { wrapper }\end{array}$ & & Provenance \\
\hline & & $\begin{array}{l}\text { Indexing materials/ technique } \\
\text { wrapper }\end{array}$ & & Language \\
\hline & & Indexing dates wrapper & & \\
\hline
\end{tabular}

Tabla IV. Relación de los esquemas de metadatos en estudio con el modelo definido para la Iglesia católica merideña (MICM).

Scire. $14: 2$ (jul.-dic. 2008) 131-149. ISSN 1135-3716. 
usar como esquema de metadatos en la descripción del patrimonio artístico del Museo Arquidiocesano de Mérida.

\subsection{Descripción de un objeto usando el CDWA}

Se ilustra con el ejemplo de la descripción de la segunda campana más antigua del mundo, la cual forma parte de la colección del Museo Arquidiocesano de Mérida

$<$ cdwalite:objectWorkTypeWrap $>$

$<$ cdwalite:objectWorkType $>$ Instrumentos musicales $</$ cdwalite:objectWorkType $>$ $<$ cdwalite:objectWorkType $>$ Campana $</$ cdwalite:objectWorkType $>$

$</$ cdwalite:objectWorkTypeWrap $>$

$<$ cdwalite:titleWrap>

$<$ cdwalite:titleSet>

$<$ cdwalite:title $>$ "Abe María" $</$ cdwalite:title $><$ cdwalite:titleSet $>$

$</$ cdwalite:titleWrap $>$

$<$ cdwalite:displayCreator $>$ Desconocido $</$ cdwalite:displayCreator $>$

$<$ cdwalite:displayMeasurements $>$ alto $42 \mathrm{~cm}$; diámetro $53 \mathrm{~cm}$

$</$ cdwalite:displayMeasurements $>$

$<$ cdwalite:displayMaterialsTech $>$ Bronce fundido $</$ cdwalite:displayMaterialsTech $>$ $<$ cdwalite:displayStateEditionWrap $>$

$<$ cdwalite:displayState $>$ Regular e incompleto $</$ cdwalite:displayState $>$

$</$ cdwalite:displayStateEditionWrap $>$

$<$ cdwalite:styleWrap>

$<$ cdwalite:style $>$ Medieval $</$ cdwalite:style $></$ cdwalite:styleWrap $>$

$<$ cdwalite:cultureWrap $>$

$<$ cdwalite:culture $>$ Española $</$ cdwalite:culture $></$ cdwalite:cultureWrap $>$

$<$ cdwalite:displayCreationDate>Finales del siglo X $(90$ ?) $</$ cdwalite:displayCreationDate $>$

$<$ cdwalite:locationWrap >

$<$ cdwalite:locationSet $><$ cdwalite:locationName type= "currentRepository" $>$ Museo Arquidiocesano de Mérida (Mérida, Venezuela) $</$ cdwalite:locationName $></$ cdwalite: locationSet>

$</$ cdwalite:locationWrap $>$

$<$ cdwalite:indexingSubjectWrap $>$

$<$ cdwalite:indexingSubjectSet>

$<$ cdwalite:subjectTerm $>$ Reverencial $</$ cdwalite:subjectTerm $>$

$<$ cdwalite:subjectTerm>Instrumentos musicales $</$ cdwalite:subjectTerm $>$

$<$ cdwalite:subjectTerm $>$ Campanas $</$ cdwalite:subjectTerm $>$

$</$ cdwalite:indexingSubjectSet $></$ cdwalite:indexingSubjectWrap $>$

$<$ cdwalite:descriptiveNoteWrap $>$

$<$ cdwalite:descriptiveNoteSet $><$ cdwalite:descriptiveNote $>$ Campana antigua de color marrón, llamada "Abe María". Forma de cono truncado o de colmena. En el cuerpo, una cruz en relieve que llega hasta la base, formada por dos barras gruesas, 
cortada por rayas oblicuas y se entrecruza por una cruz igual. Se encuentra decorada por líneas gruesas en el contorno. En la parte superior, como asidero para colgar, 4 argollas unidas en un grueso tabique central. </cdwalite:descriptiveNote> $<$ /cdwalite:descriptiveNoteSet>

$</$ cdwalite:descriptiveNoteWrap $>$

$<$ cdwalite:inscriptionsWrap >

$<$ cdwalite:inscriptions $>$ ABE MARIA GIA. PLENA AÑO DE 90_</cdwalite: inscriptions $>$

$</$ cdwalite:inscriptionsWrap $>$

$<$ cdwalite:recordWrap $>$

$<$ cdwalite:recordSource> Colección del Museo Arquidiocesano de Mérida (Mérida, Venezuela)

$</$ cdwalite:recordSource $>$

$</$ cdwalite:recordWrap $>$

Visualización del registro

Type: Instrumentos musicales

Type: $\quad$ Campana

Title: Abe María

Creator: Desconocido

Measurements: Alto $42 \mathrm{~cm}$; diámetro $53 \mathrm{~cm}$

MaterialsTech: Bronce fundido

State: Regular e incompleto

Style: Medieval

Culture: $\quad$ Española

CreationDate: Finales del siglo X (90?)

Location: $\quad$ Museo Arquidiocesano de Mérida (Mérida, Venezuela)

SubjectTerm: Reverencial

SubjectTerm: Instrumentos musicales

SubjectTerm: Campanas

DescriptiveNote: Campana antigua de color marrón, llamada "Ave María". Forma de cono truncado o de colmena. En el cuerpo, una cruz en relieve que llega hasta la base, formada por dos barras gruesas, cortada por rayas oblicuas y se entrecruza por una cruz igual. Se encuentra decorada por líneas gruesas en el contorno. En la parte superior, como asidero para colgar, 4 argollas unidas en un grueso tabique central.

Inscriptions: $\quad$ ABE MARIA GIA. PLENA AÑO DE 90

Record: $\quad$ Colección del Museo Arquidiocesano de Mérida (Mérida, Venezuela)

\section{Conclusiones}

Los métodos utilizados en la Arquidiócesis de Mérida para el registro, inventario y catalogación del patrimonio artístico están apegados a los estándares internacionales existentes, lo cual dice del cuidado que los responsables de los 
mismos han tenido para cumplir con el propósito de salvaguardar y difundir la información para las futuras generaciones y su misión evangelizadora.

Existen esquemas para la descripción de bienes culturales que pueden ser usados para la descripción e intercambio de información del patrimonio artístico de la Iglesia merideña, tales como CDWA, REACH y VRA Core, entre otros.

Con base en el estudio comparativo de los esquemas con el modelo definido para la Iglesia católica merideña concluimos que el esquema que más se adapta para el objetivo propuesto es el CDWA, por ser amplio y completo para la descripción del patrimonio artístico.

Habida cuenta de que se ha avanzado en la digitalización del patrimonio de la Iglesia merideña, el presente proyecto se centra en adoptar la metodología expuesta y el sistema escogido para aplicarlos a los bienes culturales artísticos, tal como se ha señalado a lo largo de esta investigación.

Este trabajo que ha iniciado la Arquidiócesis de Mérida es un aporte fundamental en la definición y el uso de estándares requeridos para el intercambio de información y la construcción de bibliotecas y repositorios digitales para la Iglesia católica venezolana, dada la complejidad de la estructura organizativa, el volumen y la tipología de los bienes culturales que custodia.

\section{Referencias}

Arquidiócesis de Mérida (2003). Carta Circular. // Boletín del Archivo Arquidiocesano de Mérida. VIII:23 (2003).

Bauste, Marlene; Rosales, N. Fabiola (2005). La Biblioteca Digital de la Iglesia Católica Venezolana, caso Arquidiócesis de Mérida: una propuesta para la difusión de su patrimonio en la Web. // Congreso Internacional Centenario del Archivo Arquidiocesano de Mérida, 1905-2005 (Mérida, Venezuela, 6 al 12 de noviembre 2005).

Dekkers, Makx (2000). Metadata Watch Report. 3. http://www.schemas-forum.org/ (200601-08).

Dublin Core Metadata Initiative. http://dublincore.org/ (2006-01-24).

García Cataño, Carolina; Arroyo Menéndez, David. Biblioteca Digital y Web Semántica. http://www.sindominio.net/biblioweb/telematica/bibdigwebsem.html (2006-03-12).

Getty Trust (2005-2006). CDWA Lite: specification for an XML schema for contributing records via the OAI Harvesting Protocol. http://www.getty.edu/research/conducting research/standards/cdwa/cdwalite/index.html (2006-03-18).

Gilliland-Swetland, A. J. (1998). Defining metadata. // Introduction to metadata: pathways to digital information. Los Ángeles: Getty Information Institute, 1998. 1-8.

Goncalves, M. A.; Fox, E.A.; Watson, L.T., et álii (2004). Streams, structures, spaces, scenarios, societies (5S): a formal model for digital libraries. // ACM Transactions on Information Systems. 22:2 (2004) 270-312.

Scire. 14 : 2 (jul.-dic. 2008) 131-149. ISSN 1135-3716. 
Lamarca Lapuente, María Jesús (2006). Hipertexto: el nuevo concepto de documento en la cultura de la imagen. http://www.hipertexto.info (2006-06-01).

RLG (2006). REACH element set for shared description of museum objects. http://www.rlg. org/reach.elements.html (2006-03-10).

Senso, José A.; Rosa Piñero, Antonio de la (2003). El concepto de metadato. Algo más que una descripción de recursos electrónicos. // Ciência da Informação. 32:2 (may.-ago. 2003) 95-106.

VRA Core Categories, versión 3.0. http://www.vraweb.org/vracore3.htm (2006-02-22).

Wisser, Katherine M. (ed.) (2006). North Carolina ECHO: Exploring Cultural Heritage Online. http://www.ncecho.org/Guide/toc.htm (2006-03-01).

World Wide Web Consortium Process Document (2005). http://www.w3.org/2005/10/ Process-20051014/ (2006-02-25).

W3C Recommendation. Resource Description Framework (RDF) Model and Syntax Specification (1999). http://www.w3.org/TR/1999/REC-rdf-syntax-19990222/ (2006-02-20). 